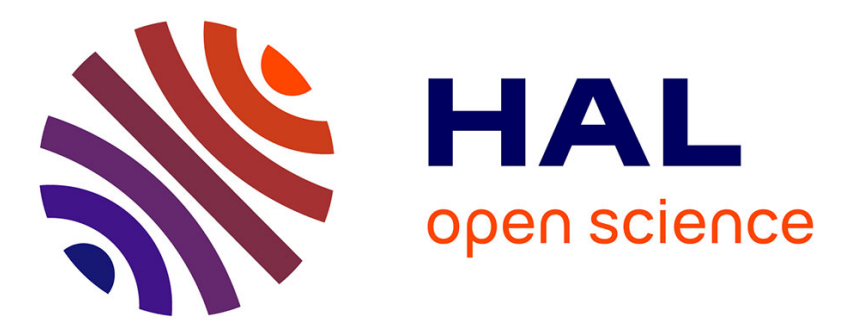

\title{
Development of a novel plane piezoelectric actuator using Hamilton's principle based model and Hertz contact theory
}

Gaston M'Boungui, Betty Semail, Frédéric Giraud, Adisa Jimoh

\section{To cite this version:}

Gaston M'Boungui, Betty Semail, Frédéric Giraud, Adisa Jimoh. Development of a novel plane piezoelectric actuator using Hamilton's principle based model and Hertz contact theory. Sensors and Actuators A: Physical , 2014, 217, pp.116-123. 10.1016/j.sna.2014.06.026 . hal-01055457

\author{
HAL Id: hal-01055457 \\ https://hal.science/hal-01055457
}

Submitted on 12 Aug 2014

HAL is a multi-disciplinary open access archive for the deposit and dissemination of scientific research documents, whether they are published or not. The documents may come from teaching and research institutions in France or abroad, or from public or private research centers.
L'archive ouverte pluridisciplinaire HAL, est destinée au dépôt et à la diffusion de documents scientifiques de niveau recherche, publiés ou non, émanant des établissements d'enseignement et de recherche français ou étrangers, des laboratoires publics ou privés. 


\title{
Development of a novel plane piezoelectric actuator using Hamilton's principle based model and Hertz contact theory
}

\author{
G M'boungui $^{1}$, B Semail ${ }^{2}$, F Giraud ${ }^{3}$, AA Jimoh ${ }^{4}$ \\ ${ }^{1,4}$ Department of Electrical Engineering, \\ Tshwane University of Technology \\ Private Bag X680, Pretoria 0001, RSA \\ ${ }^{2,3}$ Laboratoire d'Electrotechnique et d'Electronique de Puissance de \\ Lille, université Lille1 IRCICA, \\ 50 Avenue Halley, 59650 Villeneuve d'Ascq FRANCE \\ E-mail: mboungui@yahoo.fr
}

\begin{abstract}
A simple device based on friction coefficient control was designed as a solution to the lack of compactness and simplicity encountered in the number of force feedback interfaces. The structure comprises a 64 × 38 × 3 mm copper-beryllium plate on which well-adjusted polarized piezoceramics are glued. The plate stands on four legs, each of which has a spherical end. By controlling the drive voltage, friction force may be varied as required by a user who moves the device on a flat surface, as he or she would do with a normal mouse. This adds the possibility of rendering simulated forces from objects manipulated on a PC screen. Friction forces obtained using Hertz contact theory compare well with the ones measured on an experimental setup, which demonstrate the validity of the approach with regard to force feedback application
\end{abstract}

Keywords: Piezoceramic, electroactive lubrication, force feedback.

\section{Introduction}

This article explores the concept and realization of a piezoelectric actuator intended for force feedback stimulation. More precisely, the interface proposed is intended to produce a tactile interaction signal that is essentially a touch feedback mechanism. This is a sequel to a companion paper published by the authors [1] where the idea was initially introduced. Although this is an advance on that idea, it is based on a new concept.

Numerous force feedback interfaces have been developed over the years, which often utilize DC motors and entail a large number of mechanical links [2]. Subsequent to the development of the "Phantom" commercialized by Sensable®, the Pantograph, [3] a known force feedback peripheral, was developed. 
As an alternative, the action of friction generated by a surface under the finger is exploited in continuous structure tactile interfaces. Watanabee [5] pioneered friction coefficient adjustment. Watanabee used a steel beam, one end of which was attached to a Langevin transducer [6]. This transducer, which is excited at $77 \mathrm{kHz}$, communicates its maximum $2 \mu \mathrm{m}$ vibrations to the beam. As a result, the sensation experienced by a finger that explores the surface of the non-excited beam is different from the one experienced with the vibrating beam: in the latter case, the surface is very slippery and smooth. Provided vibratory amplitude and frequency conditions are satisfied, there is an overpressure or squeeze effect between a plate vibrating in front of another [7]. M. Biet [8] and L. Winfield [7] applied the same principle by replacing one of the plates by the fingertip while using the vibrating plate as an interface, with which the fingertip could interact.

The aim of this study is to design a simple 2Dof interface that is as compact as a computer mouse, but able to oppose different resistant forces whenever it is moved on a surface plane by a user, as illustrated in Figure 1. To this end, a piezoelectric actuator [4] induces the electroactive lubrication effect.

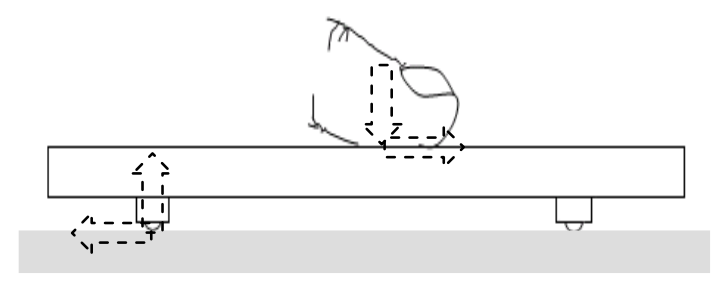

Figure 1. The concept: a plate standing on built in feet and moved by a user's finger. The dashed arrows illustrate the action of the finger and the reaction to applied forces at the contact interface respectively.

As in [6][7][8], friction coefficient adjustment remains of interest, but the fingertip alone is no longer used as a friction variation sensor, as in the devices mentioned above. In this case, the entire hand performs the sensor function. In effect, using the fingertip as a sensor may provide only limited information. M. Bouzit [25] suggested that perception in force feedback is more effective with all five fingers than using one finger only.

The design under development will consist of a thin plate with four legs, each having a spherical end on one side and piezoelectric ceramics bonded to the opposite outer face. As discussed later in the article, in this specific case, the small leg below the ultrasound vibrating piezoelectric bending actuator indents the surface below the leg, creating the controlled friction. With that indentation function of actuator vibratory parameters comprising vibratory amplitude, it is additionally the correspondence between the actuator characteristic in terms of wave amplitude response time (less than $1 \mathrm{~ms}$ ) with the range of human mechanoreceptor sensitivity and bandwidth [26] that was used to build the device. Different methods, including Finite Element Analysis (FEA) [9], have been developed to investigate such piezoelectric structures [10][11][12][13]. Recently, analysis methods and models were proposed for more complex problems in which the structure is subject to local in-plane mechanical and electrical loads [14][15], for example. 
Concerning the study of our actuator, FEA follows the modelling of the device based on Hamilton's principle. Since the study deals with a bimorph structure, taking into account the active and passive properties of its constitutive materials, the analytical approach to transcribing the bimorph vibratory behaviour is conducted by describing its energy state; its given mechanical and electrical aspects as well as the electromechanical coupling induced by piezoelectric properties. Application of the principle of stationary action (Hamiltonian), and the use of the variational method, yield the equation of mechanical equilibrium required to solve the system.

Rayleigh and Kirchhoff (Rayleigh 1894) used this energetic approach to deal with the analytical study of vibrations in membranes, beams, disks and plates, leading to equations of motion in elastic solid [16].

Firstly, the actuator is presented and then electroactive lubrication principle is described with specific reference to the particular actuator on which this study focuses. Next, the actuator's electromechanical behaviour is described. Lastly, the effectiveness of resistant force returned by the actuator is verified, in reaction to the simulated user's displacement, by means of results obtained by simulation and those from experiment.

\section{The system}

\subsection{Description}

The actuator is a mechanical amplifier consisting of a 64 x $38 \times 3 \mathrm{~mm}$ copperberyllium plate, one face of which is strongly bonded, by means of epoxy, to a matrix of PZT (P1 89) piezoceramics. These piezoceramics are $12 \times 12 \times 1 \mathrm{~mm}$, polarized and conveniently put together (Figure $2 \mathrm{a}$ ). On the opposite face (Figure $2 \mathrm{~b}$ ), four built-in legs support the plate. In addition, a measurement ceramic, utilizing the direct piezoelectric effect, is glued at the centre of the plate. The lower end of the leg (Figure 1) comprises a $1 \mathrm{~mm}$ diameter steel ball fitted in tightly.

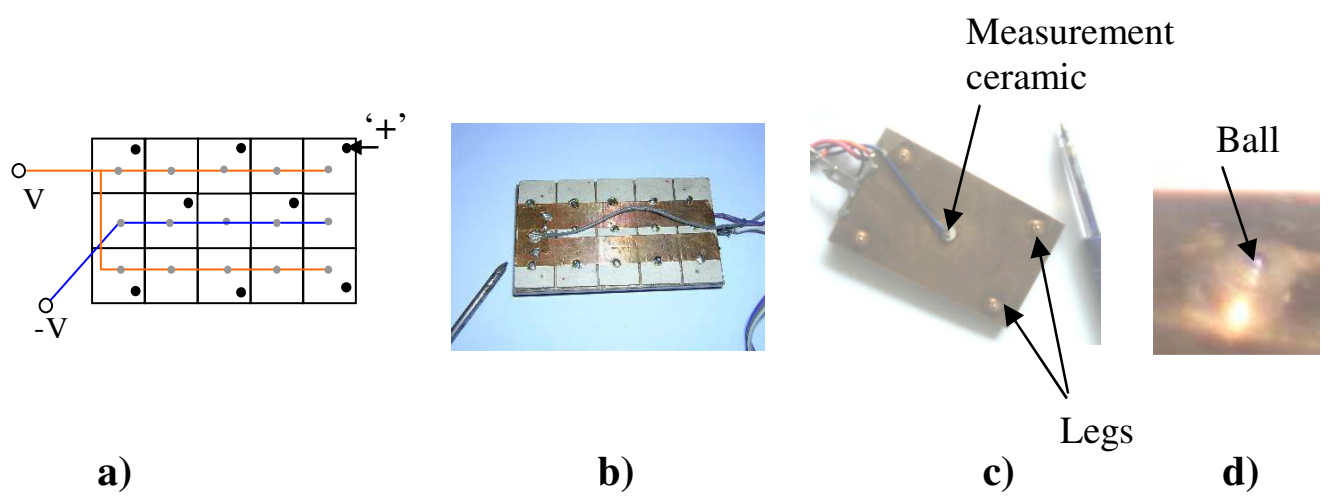

Figure 2: a) arrangement of ceramics b) plate up side c) opposite side d) leg tip

The sine voltage supply of piezoceramic electrodes creates a standing wave, sketched in Figure 3, within the plate. The legs are located at the standing wave crest so that they move normally. The leg trajectory is therefore described in Figure 3, showing several arbitrary leg positions up to the contact between the leg and the plane support, whenever the device is excited. 


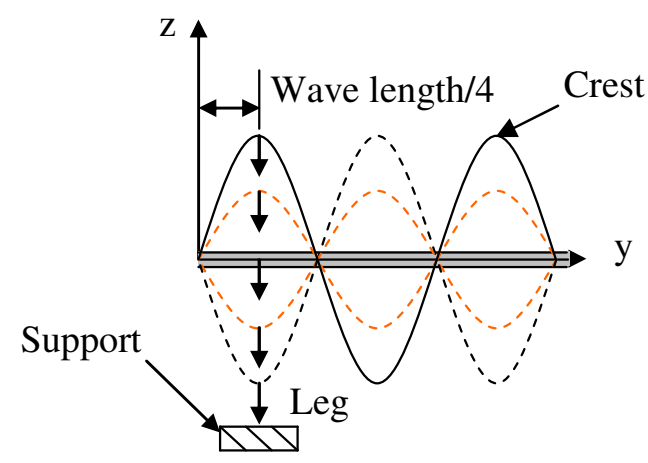

Figure 3: standing wave and foot trajectory

\subsection{Working principle}

As in Figure 4, when the user's hand holds and moves the pre-loaded actuator in the absence of a standing wave within the plate, a force is opposed to the action corresponding to Coulomb's friction law ( $F_{\text {tan gential }}=\mu F_{\text {normal }}$ ), with $\mu$ the dry friction coefficient featuring the ball - plane contact.

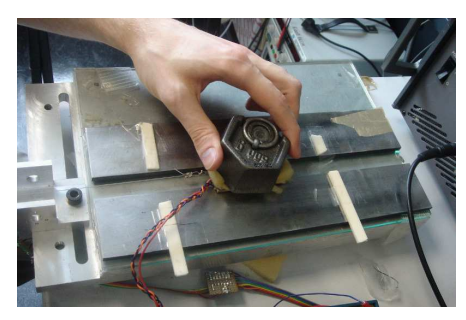

Figure 4: pre-loaded actuator, using a common weight, held and moved by the user's hand

Conversely, for certain conditions, the standing wave effect induces friction reduction between the leg tip and the surface. This is due to the appearance of intermittent contact observable at the interface of the ball and support for certain wave amplitude values. Consequently, generating and controlling the standing wave enables a variation of the friction force, in other words slip, felt by the hand.

\subsection{Electroactive lubrication}

The passive force feedback mentioned earlier utilizes the principle of electroactive lubrication presented in this section. It is a technique based upon the control of simple piezoelectric actuators which enable dry friction modulation between two solids that are dynamically in contact. Ultrasonic vibrations are the origin of the apparent friction reduction between the solids. Assuming a surface moving over another surface, let us distinguish the body's macroscopic displacement as a sum of micrometric displacements, due to the regular separation of the surfaces; it therefore becomes possible to reduce friction between the two surfaces. The micro displacements oscillating at the two surfaces subject to vibrations are source of fretting [17]. The contact occurring under those conditions may be divided into three sliding modes [18] (Figure 5). 


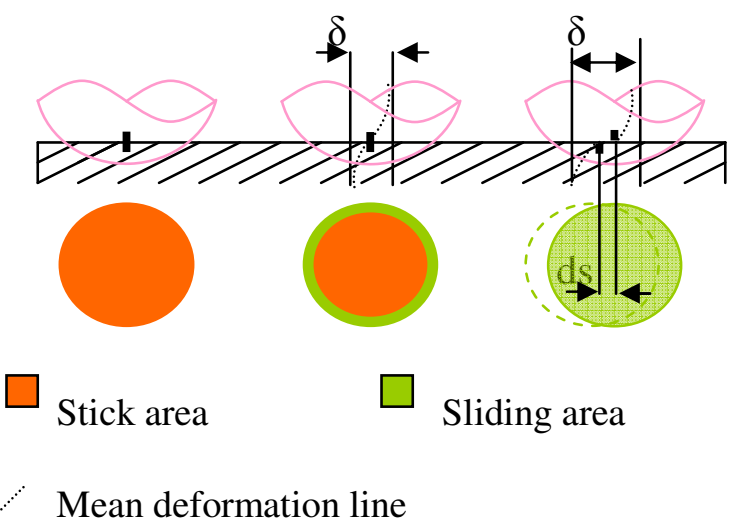

Figure 5: partial sliding- total sliding transition of sphere/plan contact

Static mode: no movement between the bodies. All material points retain their positions. As a result, there is no tangential friction force:

$$
F_{T}=0
$$

Quasi static mode or partial sliding mode: partial sliding takes place.

Despite a relative change in position, the material points of surfaces in contact do not move some with respect to others. The deformation of a few micrometers is enabled by inner material elasticity accommodation. The corresponding friction force $F_{T}$ exhibits an elastic response and partial sliding characterized by a linear relation between $F_{T}$ and the relative surface displacement $\delta$ for a normal force $F_{0}$ :

$$
F_{T}=k \delta \text { with } F_{T} \leq \mu F_{0}
$$

where $\mu$ represents the dynamic friction coefficient at the contact, and $k$ is the contact stiffness.

Total sliding: all the contact surface points move. Coulomb's Friction law applies then, in order to determine $F_{T}$ as function of $F_{0}$ :

$$
F_{T}=F_{T}^{\infty}=\mu . F_{0} \quad \forall \delta
$$

The quasi static mode offers a solution to apparent friction force reduction $F_{T}$ by limiting the distance $d_{s}$ defined in Figure 5, within each contact time along the global movement. The concept is based on separating the surfaces to limit the total sliding mode and favour partial sliding (Figure 5 refers), consequently reducing the average friction force $\left(F_{T}<F_{T}^{\infty}\right)$.

\section{Friction force modelling}

For control and simulation purposes, which are discussed in this article, the main variables influencing the displacement $d_{s}$ that will constitute the force feedback device inputs are highlighted. 
To this end, Hertz' theory [4] provides the analytic relationships describing those systems for elementary geometries. In the instance of sphere/plane geometry, a stiffness function $\mathrm{R}[18]$ is defined to express the penetration depth $e$ resulting from the applied normal force $F_{d}$.

In the presence of a static normal load $F_{0}$, the ball indents the plane of a depth $e_{0}$ :

$$
e_{0}=R\left(F_{0}\right)=\sqrt[3]{\frac{. F_{0}^{2}}{K^{2}}}
$$

With

$$
K=\frac{4}{3} \frac{\sqrt{\Phi / 2}}{\frac{1-v_{1}^{2}}{E_{1}}+\frac{1-v_{2}^{2}}{E_{2}}}
$$

$\mathrm{E}_{\mathrm{i}}$ is the Young modulus of the considered material $\mathrm{i}$ while $v_{\mathrm{i}}$ is the Poisson coefficient; $\mathrm{i}=1,2$. $\Phi$ represents the diameter of the ball.

Imposing a vibration $y$ on the contact leads to a dynamic force $F_{d}$ that may be expressed as:

$$
\begin{aligned}
& F_{d}(t)=R^{-1}\left(R\left(F_{0}\right)+W \cos (\omega t)\right) \\
& y(t)=W \cos (\omega t)
\end{aligned}
$$

If the absolute value of the over-imposed amplitude $W$ is greater than the static indentation $e_{0}$, there is surface dissociation. That is:

$$
W \geq R\left(F_{0}\right)
$$

The distance of slipping within two successive instants $t_{1}$ and $t_{2}$ is obtained by integrating the speed $v$ as follows:

$$
\begin{aligned}
d s & =\int_{t 1}^{t 2} v(t) d t \text { with } F_{d}\left(t_{1}, t_{2}\right)=0 \\
t_{1} & =\frac{1}{\omega} a \cos \left(\frac{R^{-1}\left(F_{0}\right)}{W}\right) \text { and } t_{1}+t_{2}=\frac{2 \pi}{\omega}
\end{aligned}
$$

Equation (8) demonstrates that for a given value of $\omega$ (angular frequency), an increase in the vibratory amplitude $W$ results in a reduction of the slip distance $d_{s}$. This creates partial slip conditions and gives advantage to the reduction of the average friction coefficient $\mu_{0}$.

$\mu_{0}$ is defined as the average value of friction coefficient over a period. It corresponds to the ratio $F_{d} / F_{0}$ which may be less than the static friction coefficient, depending on excitation conditions. 


\section{Electromechanical behaviour of the actuator}

FEA was utilized to benefit from 3D animations and obtain insights regarding the behaviour of the plate. However, it will prove of interest to predict the plate mechanical displacement patterns and modal frequencies using a known analytical approach, which ultimately permits us to reaffirm the displacements of the legs' tips. We therefore summarize the chosen approach, firstly, in this section. In fact, the plate behaviour is interpreted through the application of the principle of stationary action (Hamiltonian) and the use of a variational method: the Oxyz frame and median plane as sketched in Figure 6. This yields the bimorph (a thin plate) equation of mechanical equilibrium; it is briefly discussed. The approach is inspired by the work of [19].

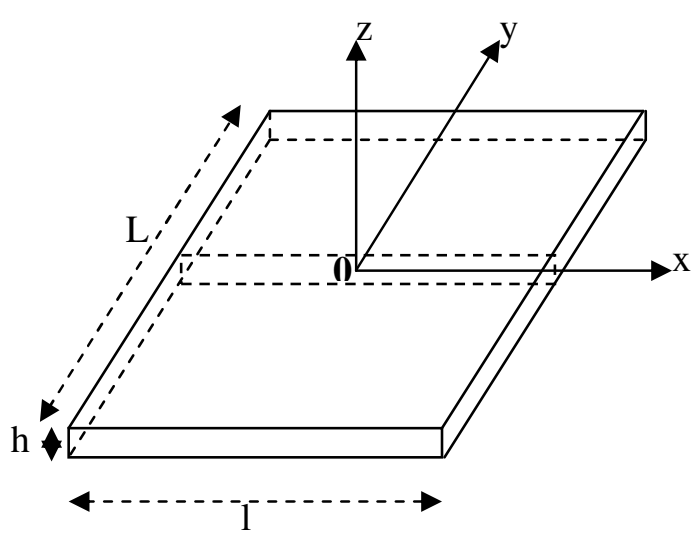

a)

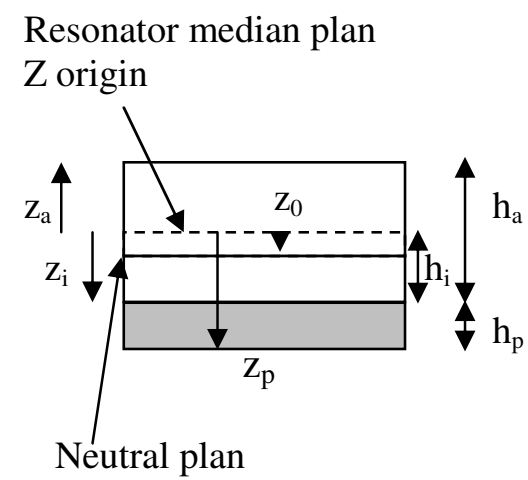

b)

Figure 6: a) (Oxyz) definition b) median plane definition

In Figure 6, $L$ and $l$ respectively denote the plate length and width, $h_{p}$ and $h_{a}$ represent the ceramic and resonator thickness; $z_{a}$ is the resonator upper plane ordinate; $z_{p}$, the piezoceramic lower plane ordinate and $z_{i}$, the ordinate of the common plane to the two materials.

A generalized form of Hamilton's principle for the coupled electromechanical system is presented as [19]:

$$
\partial \int_{t_{1}}^{t_{2}} \ell d t=0
$$

where $\ell$ is the Lagrangian of the system that may be expressed as:

$$
\ell=\frac{1}{2} \int_{\Sigma}\left(\int_{z_{p}}^{z_{i}}\left(\rho_{p} \dot{U}^{t} \dot{U}-S^{t} T_{p}\right) d z+\int_{z_{i}}^{z_{a}}\left(\rho_{i} \dot{U}^{t} \dot{U}-S^{t} T_{i}\right) d z\right)+W_{\text {sources }}
$$

In Equation (10), $W_{\text {sources }}$ is the pseudo-potential accounting for the work furnished by external sources; $\rho$ represents the density with indices $i$ and $p$ respectively denoting the piezoelectric and the isotropic materials. $U$ gives the vector of mechanical displacements while $S$ and $T$ respectively represent the stress and strain. 
Since the implementation is similar to [19], only the details necessary for applying the method to the specific problem are presented here. Using assumptions proposed by Kirchhof, related to plate shear deformation [19], the set of displacements is firstly furnished:

$$
\begin{aligned}
& u=u_{0}(x, y)-z \frac{\partial w}{\partial x} \\
& v=v_{0}(x, y)-z \frac{\partial w}{\partial y} \\
& w=w(x, y)
\end{aligned}
$$

that is, $\boldsymbol{U}(u, v, w)$ where the known displacements $u_{0}(x, y)$ and $v_{0}(x, y)$ result from the plate pre-extension following an in-plane pre-stress. Next, for the sake of convenience, the strains are calculated according to Green's method [20] so that the strains tensor is expressed as follows:

$$
S_{i j}=\frac{1}{2}\left(\frac{\partial u_{i}}{\partial x_{j}}+\frac{\partial u_{j}}{\partial x_{i}}+\frac{\partial u_{m}}{\partial x_{i}} \frac{\partial u_{m}}{\partial x_{j}}\right)=\frac{1}{2}\left(u_{i, j}+u_{j, i}+u_{m, j} . u_{m, i}\right)
$$

After development, and taking the Kirchhof assumptions mentioned earlier into account while noting the partial derivative as $f_{, i}=\frac{\partial f}{\partial x_{i}}, f_{, i i}=\frac{\partial^{2} f}{\partial x_{i}^{2}}$ and omitting the second order components:

$$
S=\left(\begin{array}{l}
\left(z_{0}-z\right) w_{, x x} \\
\left(z_{0}-z\right) w_{, y y} \\
\left(z_{0}-z\right) w_{, x y}
\end{array}\right)
$$

The stress $T$ - strain $S$ relation is obtained applying Hooke's law ( $T=H S$ ) to the assumed linear and isotropic material. It can be shown that

$$
H=\frac{E_{i}}{1-v_{i}^{2}}\left(\begin{array}{ccc}
1 & v_{i} & 0 \\
v_{i} & 1 & 0 \\
0 & 0 & \left(1-v_{i}\right)
\end{array}\right)
$$

$E_{i}$ denotes the Young modulus and $v_{i}$ the Poisson coefficient $(i=1,2)$ of the considered material.

In the piezoelectric material, by retaining the relevant terms in the constitutive equation, the stresses and strains can be related:

$$
\left.\left.\begin{array}{l}
S=s^{E} T+d^{t} E \\
D=d T+\varepsilon^{T} E
\end{array}\right\} \quad \text { or } \quad \begin{array}{l}
T=c^{E} S-e^{t} E \\
D=e S+\varepsilon^{S} E
\end{array}\right\}
$$


with

$$
c^{E}=\left[s^{E}\right]^{-1} \text { and } e=d\left[c^{E}\right]
$$

In equation (15), $E$ and $D$ represent the vectors of electric field and electric displacement respectively. As illustrated in Figure 2 a) the voltage is set to zero on the electrode in contact with the copper-beryllium while the voltage on the free electrode segment is $V$. Noting $w(x, y, t)$, the average vertical displacement and $h_{p}$ the ceramic thickness, the application of free ends boundary conditions yields [22] $E=\left(0,0,-V(t) / h_{p}\right)$ assuming the flexion effect is neglected. It can therefore be proven that:

$$
T_{p}=\left(\begin{array}{l}
c_{11}^{E}\left(z_{0}-z\right)\left(w_{, x x}+v_{p} w_{, y y}\right)+e_{31} \frac{V}{h_{p}} \\
c_{11}^{E}\left(z_{0}-z\right)\left(v_{p} w_{, x x}+w_{, y y}\right)+e_{31} \frac{V}{h_{p}} \\
c_{66}^{E}\left(z_{0}-z\right) w_{, x y}
\end{array}\right) .
$$

Finally, from equation $D=e S+\varepsilon^{S} E$, it follows that

where

$$
D=\left(0,0, e_{31}\left(z_{0}-z\right)\left(w_{, x x}+w_{, y y}\right)-\varepsilon_{33}^{S} \frac{V}{h_{p}}\right)
$$

$$
\varepsilon_{33}^{S}=\varepsilon_{33}^{T}\left(1-2 \frac{d_{31}^{2}}{\varepsilon_{33}^{T}\left(s_{11}^{E}+s_{12}^{E}\right)}\right)
$$

It turns out that all the ordinates are taken with respect to the $z_{0}$, ordinate of the neutral axis, its value is required of which the expression is [19][21]:

$$
\begin{aligned}
& z_{0}=-\frac{1}{2}\left(h_{i}+h_{p}\right) \alpha \\
& \alpha=\frac{\left(v_{i}+1\right)\left(v_{p}-1\right) C_{11}^{E} h_{p}}{C_{11}^{E} h_{p}\left(v_{i}+1\right)\left(v_{p}-1\right)-E_{i} h_{i}}
\end{aligned}
$$

The thickness between coordinates $z_{0}$ and $z_{i}$ is represented by $h_{i}$ in equation (20). Since derivation and integration are commutative operations, one can write

$$
\delta A=\delta \int_{t_{1}}^{t_{2}} \ell d t=0 \Leftrightarrow \int_{t_{1}}^{t_{2}} \delta \ell d t=0
$$

This leads to the overall material equation of equilibrium:

$$
M_{b} \ddot{w}+D_{b} \Delta^{2} w-\Delta M_{V}-p=0
$$

with $M_{b}\left[\mathrm{~kg} / \mathrm{m}^{2}\right]$ the surface mass of the bimorph: 


$$
M_{b}=\int_{p}^{i} \rho_{p} d z+\int_{z_{i}}^{z_{a}} \rho_{i} d z=\rho_{p} h_{p}+\rho_{i} h_{a}
$$

$D_{b}[\mathrm{Nm}]$ the bending stiffness of the plate:

$$
\begin{aligned}
& D_{b}=D_{a}+D_{p} \\
& D_{a}=\frac{E}{1-v_{i}^{2}} h_{a}\left(\frac{h_{a}^{2}}{12}+\alpha^{2} d^{2}\right) \\
& D_{p}=c_{11}^{E} h_{p}\left(\frac{h_{p}^{2}}{12}+(1+\alpha)^{2} d^{2}\right)
\end{aligned}
$$

and $M_{v}$ the bending moment:

$$
M_{V}=k_{b} V
$$

$k_{b}[\mathrm{~N} / \mathrm{V}]$ or $[\mathrm{C} / \mathrm{m}]$ denotes the electromechanical coupling factor:

$$
k_{b}=-e_{13}(1+\alpha) \frac{h_{a}+h_{p}}{2}
$$

In equation (23), $\Delta^{2}=\Delta \circ \Delta$ whereas $p$ represents the external loads applied on the external face of the plate that are considered null in this specific case. Assuming a harmonic movement and focusing on the $y$ axis, that is the plate length, $w(y, t)=w(y) \sin \omega t$, Equation (23) becomes:

$$
-\omega^{2} M_{b} w+D_{b} \Delta^{2} w=0
$$

Further, for a non-preloaded plate of bending rigidity $E I$ and unit length mass $m$ considered constant along the length, the Eigen-vibrations equation is reduced to [21]:

$$
\frac{d^{4} w}{d y^{4}}-\omega^{2} \frac{m}{E I} w=0
$$

Writing $\quad \lambda^{4}=\frac{\omega^{2} m L^{4}}{E I}$,

Equation (31) may be solved to derive $\lambda$ corresponding to the $6^{\text {th }}$ mode, for example. 'mode' means the number of the half bending waves along the y axis

Thus the normalized wave shape follows ([22][16]): 


$$
\begin{aligned}
& w(y)=A_{y}\left[-\operatorname{sh}\left(\lambda\left(\frac{1}{2}-\frac{y}{L}\right)\right)+\operatorname{ch}(\lambda) \sin \left(\lambda\left(\frac{y}{L}+\frac{1}{2}\right)\right)-\cos (\lambda) \operatorname{sh}\left(\lambda\left(\frac{y}{L}+\frac{1}{2}\right)\right)+\ldots\right. \\
& \left.\ldots \sin \left(\lambda\left(\frac{1}{2}-\frac{y}{L}\right)\right)-\operatorname{sh}(\lambda) \cos \left(\lambda\left(\frac{y}{L}+\frac{1}{2}\right)\right)+\sin (\lambda) \operatorname{ch}\left(\lambda\left(\frac{y}{L}+\frac{1}{2}\right)\right)\right]
\end{aligned}
$$

with $\mathrm{A}_{\mathrm{y}}$ defined so that $|w(y)=1|$ :

$$
A_{y}=-2\left(\left(\operatorname{ch}\left(\frac{\lambda}{2}\right)+\cos \left(\frac{\lambda}{2}\right)\right) \cdot\left(-\sin \left(\frac{\lambda}{2}\right) \operatorname{ch}\left(\frac{\lambda}{2}\right)+\operatorname{sh}\left(\frac{\lambda}{2}\right) \cos \left(\frac{\lambda}{2}\right)\right)\right)^{-1} .
$$

Figure 6 illustrates the shape of the neutral plan strain according to Eigen-mode $(0,6)$; $y \in\left[-\frac{L}{2}, \frac{L}{2}\right]$.

The FEA was performed using commercially available Abaqus ${ }^{\circledR}$ software. The plate was modelled with 3D cubic elements (C3D8, C3D8E) which were suitable for modelling the piezoelectric properties mentioned earlier. More precisely, the beryllium and lead zirconate titanium (PZT), namely P1 89 properties, were taken into account for the respective layers in the model as well as the ceramics' polarization. The bonding layer was omitted and the FEA showed a corresponding resonant wave shape and frequency equal to $42.7 \mathrm{kHz}$.

Table 1. Material properties

\begin{tabular}{|lll|}
\hline & Beryllium & PZT \\
\hline Young's modulus [GPa] & 123 & 72.6 \\
\hline Poisson ratio & 0.3 & 0.31 \\
\hline Mass density $\left[\mathbf{1 0}^{\mathbf{3}} \mathbf{~ k g} \mathbf{m}^{\mathbf{3}}\right]$ & 8.25 & 7.7 \\
\hline Elements & C3D8 & C3D8E \\
\hline Material Property & & Orthotropic \\
\hline
\end{tabular}

The PZT properties in Table 1 are completed with Elasticity $\left[\mathrm{N} / \mathrm{m}^{2}\right]$, Piezoelectric coupling (stress coefficients) [Coulomb/m2] and Dielectric [Farad $/ \mathrm{m}]$ matrices, given in the Appendix.

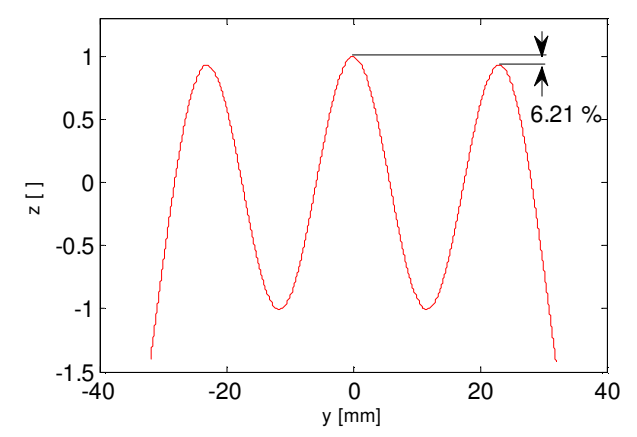

a)

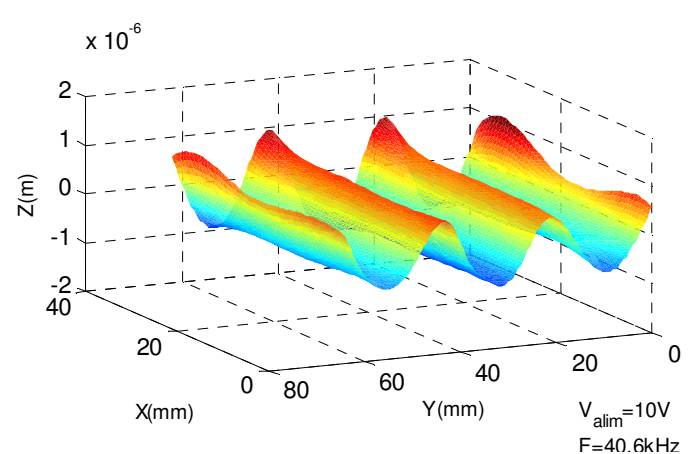

b) 


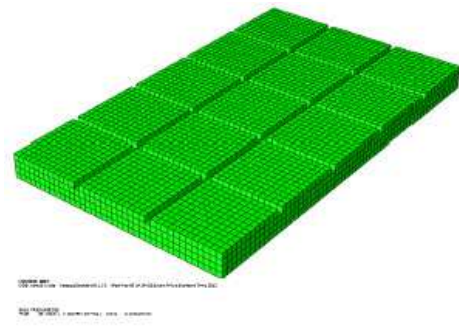

c)

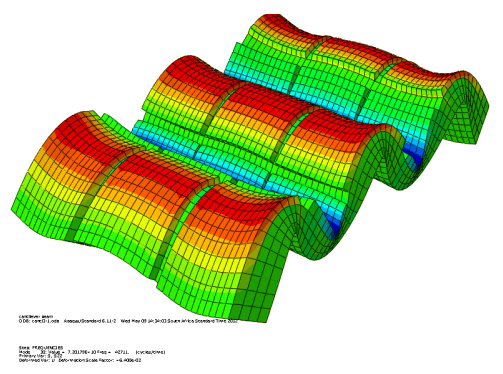

d)

Figure 7: mode $(0,6)$ a) theoretical normalized wave shape b) experimental wave shape c) Finite Element geometric model d) plate wave shape with magnitude corresponding to an arbitrary value.

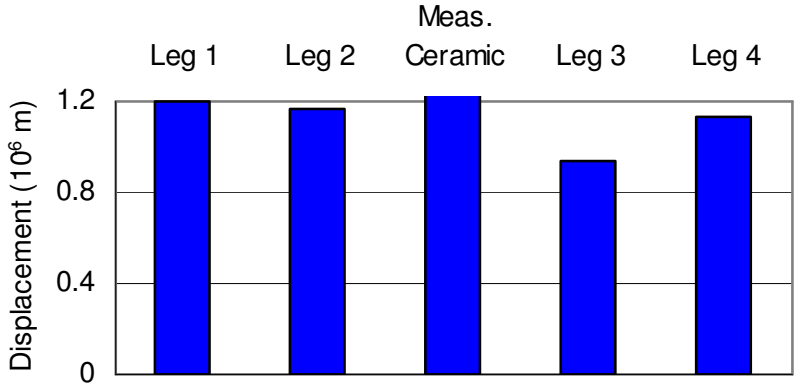

a)

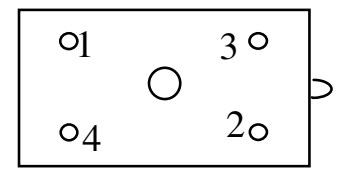

b)

Figure 8: a) maximum displacement at legs' ends b) leg mark $(1,2,3$, and 4) in the plate; the circular shape in the centre representing the measurement ceramic.

Figure $7 \mathrm{~b}$ ) depicts the mapping of the plate deformation measured using a Doppler Laser vibrometer (OFV-505, Polytech GmbH) connected to a controller OFV-5000. The controller is in its turn connected to an oscilloscope. The resonant frequency of $40.6 \mathrm{kHz}$ is acceptable compared to the $42.7 \mathrm{kHz}$ from FEA. The observed relative error of $4.9 \%$ may be explained by omission of an epoxy layer, damping, etc. in the model.

It is of importance to pay particular attention to the legs, since their displacements depend upon the accuracy of their positioning with respect to the wave crests. Since this positioning accuracy, as mentioned earlier, has a greater effect on the overall behaviour of the structure, measurement of the vibratory amplitude is made at each leg tip and also at the centre of the measurement ceramic, in plate resonant frequency conditions. The results are indicated in Figure 8 a).

As anticipated, it was found that the highest displacement is from the measurement ceramic centre while the displacement from the legs is lower. This concurs with the findings depicted in Figure 7 a) although some discrepancies were observed; Figure 8 a) revealed that, for three legs out of the four, the gap between the maximum vibratory amplitude at leg tip and that taken at the geometric centre of the plate where the measurement electrode is located, was $8.87 \%$. Looking at the displacement of the 
neutral plane in figure 7 a), the theoretical value compares adequately enough with the experimental one $(8.87 \%)$, since it is $6.61 \%$.

To prevent discrepancies that would come from gluing, the legs were built into the plate. This means that the irregularity noticed at leg number 3 may have come from inaccurate positioning of the leg or the ball with respect to the wave crest.

\section{Control of the vibration amplitude}

From the preceding paragraphs, the standing wave amplitude appears to be a key point for friction tuning at the leg /support interface.

Control of the vibratory amplitude may be achieved utilizing different approaches. In [23], the wave amplitude control is carried out by the phase control of the standing wave, according to the voltage signal supply. The advantage of this method is robustness against resonance frequency variations. One drawback is a lower dynamic behaviour due to the response time imposed by a phase locked loop (PLL).

Another way to control the wave amplitude is to tune the supply frequency around the resonance value. This approach is based on the characteristic frequency - vibratory amplitude which reveals that beyond the resonant frequency, the wave amplitude $\mathrm{W}$ decreases quasi-linearly, making its control possible [24]. The method presents the advantage of being easy to implement while the loop dynamic is rapid. Conversely, it has the disadvantage that changes in temperature displace the resonant frequency and lead to discrepancies in the control. To avoid errors, an algorithm to track the resonant frequency should be rigorously implemented to anticipate the preload influence on the resonant frequency. Nevertheless, this approach was chosen as it is also easy to implement.

At this point, an experiment was carried out to check the dynamic response time of the plate as well as the accuracy of the wave amplitude control, which is briefly discussed in the next section. In Figure 9, the plate response time, in use with a preload on it, is approximately $1 \mathrm{~ms}$. Thus, according to the human mechanoreceptor sensitivity and band-width [26], the changes induced on the friction force by the wave amplitude evolution will be readily sensed by the hand.

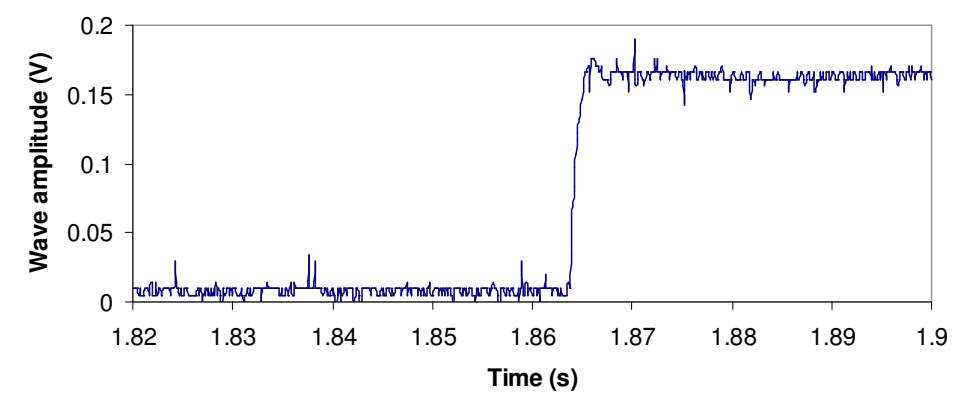

Figure 9: wave amplitude response to a unit step reference.

\section{Features of friction forces}

Prior to studying the aptitude of the device to force feedback, characterization of the friction forces at play in reaction to displacement imposed by the user was established 
by the measurement of these forces. To this end, for different vibratory amplitudes, a DC motor ${ }^{\circledR}$ Maxon controlled in speed to which the plate is attached by means of an inextensible cable and a $10 \mathrm{~mm}$ diameter pulley is used. The measured motor current is therefore a reflection of the torque and thus the force developed by the motor. That force is therefore equal in absolute value to that of the explored friction force.

An optical encoder measured the motor rotational speed and the so-constituted setup was controlled by a dSPACE DS1104 application. Several simulations based on the Hertz sphere - plane, described earlier, were performed and the results, which were compared to the experiment, are depicted in Figure 10.

Regarding the simulations mentioned above, from the equations introduced in section 3 , it was possible to compute the behaviour of the actuator for a given wave amplitude, a given tangential speed and a given normal load. The results obtained are as indicated in Figure 10.

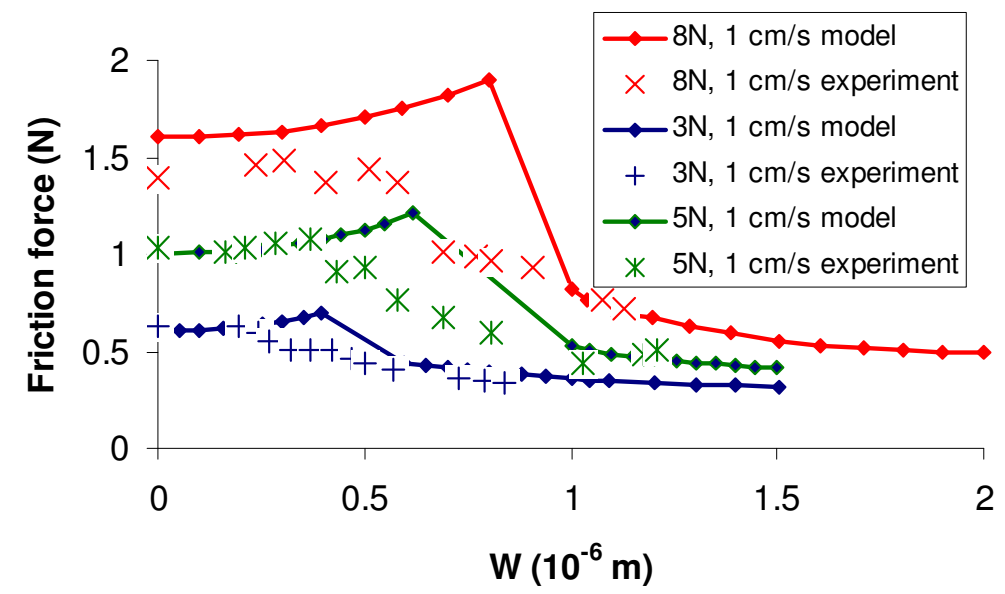

a)

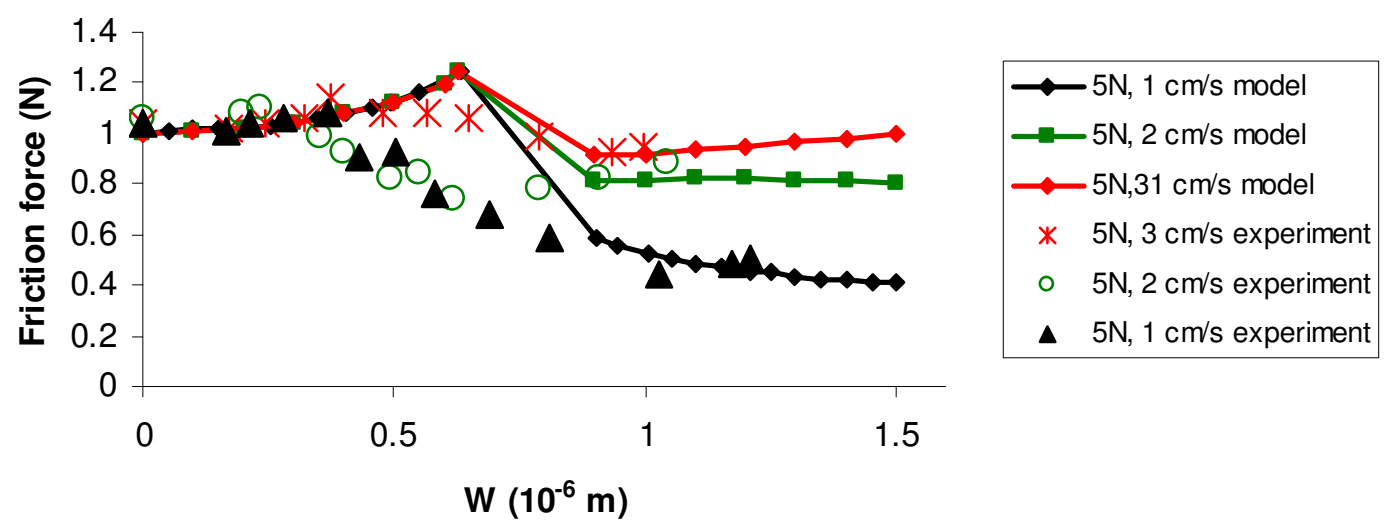

b)

Figure 10: friction force on the plate for a) a fixed preload as a function of vibratory amplitude and arbitrary tangential displacement speed, b) a given tangential speed as a function of the wave amplitude and the normal pre-load.

The experimental results presented in Figure 10 were obtained in such a way that a load $M_{\text {ext }}$ was applied on the top of the device to assume pre-stress. This load lay on 
an elastic element the stiffness of which was low enough to consider the force $F_{0}$ due to $M_{\text {ext }}\left(F_{0}=9.81 M_{\text {ext }}\right)$, constant. In addition, a steel substrate $(\mu=0.2)$ was used for these trials. Finally, the time variable displacement $w$, was imposed by the plate vibrations.

In Figure 10, several discrepancies are observed between theoretical and experimental values: 1) in friction force magnitude in the case of $8 \mathrm{~N}$ preload, for example; 2) at transition point (corresponding to $\mathrm{W}_{\text {transit. }}$ in Figure $10 \mathrm{~b}$ )), where the friction force starts to decrease. In reality, the experimental critical vibratory amplitude (transition point in Figure $10 \mathrm{~b}$ )), is always less than the theoretical one, particularly when the applied normal force is increased. This, on the one hand, may to a certain extent be explained by the assumption made when considering the ball's elasticity only. On the other hand, the contact ball - leg (ball assembled tightly into the leg) was not perfect; furthermore that contact irregularities were noticed for the four feet. The explanation may also concern an additional mechanical phenomenon. Despite this, it is notable that overall, these curves do indicate that there is a similarity that exists between the theoretical and experimental results. In particular, the friction force reduction occurs from certain vibratory amplitude corresponding to a value of transition characteristic of intermittent contact between the leg and the surface. For higher vibratory amplitudes, the contact is undeniably intermittent and enables a reduction in the friction force function of the actuator displacement speed. More precisely, in Figure 10 a) it may be noticed that the reduction reaches $59.0 \%$ (passage from $1.07 \mathrm{~N}$ to .44 $\mathrm{N}$ for a preload of $5 \mathrm{~N}$ at $1 \mathrm{~cm} / \mathrm{s}$ ). Elsewhere, for low vibratory amplitudes, the tangential force is not reduced, but greatly increased. Then, beyond the critical vibratory amplitude $\left(\mathrm{W}_{\text {transit }}\right)$, it decreases again. The reason for this may be the increase of the dynamic load $\mathrm{F}_{\mathrm{d}}$.

From Figure $10 \mathrm{~b}$ ), it appears that the tangential speed has considerable high impact upon the friction reduction. In particular, the instance of a speed equal to $3 \mathrm{~cm} / \mathrm{s}$ is observed. In this specific case, beyond critical vibratory amplitude, the tangential force increases again, prefiguring viscous type behaviour. Moreover, it is noticed that the speed of exploration should not be increased at will, since at relatively high speeds the tangential force is no longer significantly reduced (maximum of approximately 10 $\%$ reduction for $5 \mathrm{~N}$ preload; $3 \mathrm{~cm} / \mathrm{s}$ speed).

Lastly, the control of the device has been presented in 1D operation mode, largely because of the structure of the experimental setup and the rotating optical position sensor that was used. Nevertheless, these experiments could be extended into a second direction for operation as a $2 \mathrm{D}$ device by using a $2 \mathrm{D}$ position sensor.

\section{Conclusion}

The concept of a force feedback interface, based on friction coefficient reduction, is presented in the article. The redesigning of a 2 Dof passive device, which was originally designed for translation purposes, allowed the researchers to propose a promising simple and compact structure. Once the structure had been described, the reader is reminded the electroactive lubrication principle. In effect, by controlling the parameter $\mathrm{W}$, the vibratory amplitude of the standing wave generated in the plate, 
friction is effectively adjusted between the plate and the substrate to vary the simulated force felt by a user displacing the plate.

The work done so far is a preliminary stage for implementing a new device able to mimic sensations rendered by exploration of virtual surfaces. Furthermore, a focus on a more accurate dynamic prediction of the system, using FEA, will lead to improvement of the performance of the actuator which will have the spherical shape machined in the leg tip; a system to be assessed by a suitable experimental psychophysical protocol.

\section{Appendix}

Elasticity matrix:

$\left[\begin{array}{cccccc}15.37 & 8.23 & 8.06 & 0 & 0 & 0 \\ & 15.37 & 8.06 & 0 & 0 & 0 \\ & & 13.74 & 0 & 0 & 0 \\ & & & 4.59 & 0 & 0 \\ & & & & 4.59 & 0 \\ & & & & & 3.57\end{array}\right] 10^{10} \mathrm{Nm}^{-2}$

Piezoelectric coupling matrix

$$
\begin{aligned}
& d=\left[\begin{array}{cccccc}
0 & 0 & 0 & 0 & 280 & 0 \\
0 & 0 & 0 & 0 & 280 & 0 \\
-108 & -108 & 240 & 0 & 0 & 0
\end{array}\right] 10^{-12} C N^{-1} \text { or } \\
& e=\left[\begin{array}{cccccc}
0 & 0 & 0 & 0 & 12.85 & 0 \\
0 & 0 & 0 & 0 & 12.85 & 0 \\
-6.14 & -6.14 & 15.56 & 0 & 0 & 0
\end{array}\right] \mathrm{Cm}^{-2}
\end{aligned}
$$

Dielectric matrix:

$$
\left[\begin{array}{lll}
13.7 & & \\
& 13.7 & \\
& & 10.17
\end{array}\right] 10^{-9} \mathrm{Fm}^{-1}
$$

\section{References}

[1] G. M'Boungui, B. Lemaire-Semail, F. Giraud, Piezoelectric actuator for a forcefeedback application: Preliminary evaluation, World Haptics Conference (WHC 2009), pp.85-90, Third Joint EuroHaptics Conference and Symposium on Haptic Interfaces for Virtual Environment and Teleoperator Systems, 2009. 
[2] C. Schneider, T. Mustafa, A. M. Akamura, A magnetically-actuated friction feedback mouse, In: Proceedings of EuroHaptics 2004, Munich, Germany, pp. 330-337, 2004.

[3] G. Campion, Q. Wang, V. Hayward, The Pantograph Mk-II: A Haptic Instrument, 2005 IEEE/RS, Proc. IEEE/RSJ Int'l Conf. Intelligent Robots and Systems (IROS 2005), pp. 723-728, 2005.

[4] L. Garbuio, J.F. Rouchon, Etude du débrayage des moteurs piézoélectriques à rotation de mode, RIGE, Vol. 7:3-4, pp.297-314, 2004.

[5] T. Watanabe, S. Fukui, A method for controlling tactile sensation of surface roughness using ultrasonic vibration, 1995 IEEE Int. Conf. on Robotics and Automation, pp 1134-1139, 1995.

[6] M. Budinger, J.F. Rouchon, B. Nogarede, Analytical modelling for the design of a piezoelectric rotating-mode motor, IEEE/ASME Transactions on Mechatronics, Vol. 9:1, pp.1-9, 2004.

[7] L. Winfield, J. Glassmire, J. E. Colgate, M. Peshkin, T-PaD: Tactile pattern display through variable friction reduction, Second Joint Eurohaptics Conference and Symposium on Haptic Interfaces for Virtual Environment and Teleoperator Systems (WHC), 2007.

[8] M. Biet, F. Giraud, B. Semail, Squeeze film effect for the design of an ultrasonic tactile plate, In: IEEE Transactions on Ultrasonic, Ferroelectric and Frequency Control, Volume: 54, Issue: 12, pages: 2678 - 2688, December 2007.

[9] C. Poizat and A. Benjeddou, On analytical and finite element modelling of piezoelectric extension and shear bimorphs, Computers and Structures, Vol. 84, pp. 1426-1437, 2006.

[10] M. Goldfarb and N. Celanovic, Modelling piezoelectric stack actuators for control of micromanipulation, IEEE Control Systems, Vol. 17, no. 3, pp. 69-79, June 1997.

[11] H. Adriaens, W. L. de Koning, and R. Banning, Modelling piezoelectric actuators, IEEEIASME Transactions on Mechatronics, Vol. 5, p. 331, 2000.

[12] A. Fernandes and J Pouget, An accurate modelling of piezoelectric multi-layer plates, European Journal of Mechanics AlSolids, Vol. 21, pp. 629-651, 2002.

[13] N. W. Hagood, W. Chung, A. von Flotow, Modelling of piezoelectric actuator dynamics for active structural control, Journal of Intelligent Material, Systems and Structures, Vol.1:3, pp. 327 - 354, 1990.

[14] X. D. Wang and G. L. Huang, Wave propagation generated by piezoelectric actuators attached to elastic substrates, Acta Mechanica, 183: 155-176, 2006.

[15] C.R. Jin and X.D. Wang, Analytical modelling of the electromechanical behaviour of surface-bonded piezoelectric actuators including the adhesive layer, Engineering Fracture Mechanics, 78:2547-2562, 2011. 
[16] M. Géradin, D. Rixen, Théorie des vibrations. Masson, Paris, 1993.

[17] K.L. Johnson, 1985. Contact mechanics, Cambridge University Press.

[18] L. Garbuio, F. Pigache, J.F. Rouchon, B. Semail, Ultrasonic friction drive for passive force feedback devices, Electromotion, Vol.14:2, April-June 2007.

[19] C. Giraud-Audine, B. Nogarède, Analytical modelling of travelling-wave piezomotor stators using a variational approach, The European Physical Journal of Applied Physics, Vol. 6: 01, pp. 71-79, April 1999.

[20] J. Courbon, Théories de l'élasticité, Les Techniques de l'Ingénieur, AF5(A305), Août, 1979.

[21] M. Géradin and D. Rixen, Théorie des vibrations. Masson, Paris, 1996.

[22] F. Galiano, Conception, modélisation théorique et Caractérisation des Moteurs Piézoélectriques Mono et Multi degrés de Liberté: Applications á la Microrobotique et aux Micromachines, PhD Thesis, LEEI de Toulouse, ENSEEIHT, No. 536, November, 2000.

[23] F. Pigache, B. Lemaire-Semail, F. Giraud, A. Bouscayrol, Control of a piezoelectric actuator for adjustable brake in haptic devices , EPE 05, DRESDE, 11-14 September 2005, CD-ROM ISBN 90-75815-08-05.

[24] E. Piecourt, M. Lajoie Mazenc, Electromechanical characterization and power supply of piezoelectric motors, $\mathrm{PhD}$ thesis, Institut National Polytechnique de Toulouse, Toulouse, France, No. 95, INPT 0102, 1995.

[25] M. Bouzit, G. Burdea, G. Popescu, R. Boian, The Rutgers Masters II- New Design Force - Feedback Glove. IEEE/ASME Transactions on Mechatronics, Vol. 7:2, pp. $256-263,2002$.

[26] D. Pawluk, R. Howe, A holistic model of human touch, submitted to the $5^{\text {th }}$ Annual C.N.S. Meeting, Boston, MA 1996. 